\title{
Pairing mechanism of high-temperature superconductivity: Experimental constraints
}

\author{
Guo-meng Zhao ${ }^{1,2, *}$ \\ ${ }^{1}$ Department of Physics and Astronomy, California State University, Los Angeles, CA 90032, USA \\ ${ }^{2}$ Department of Physics, Faculty of Science, Ningbo University, Ningbo, P. R. China
}

\begin{abstract}
Developing a theory of high-temperature superconductivity in copper oxides is one of the outstanding problems in physics. It is a challenge that has defeated theoretical physicists for more than twenty years. Attempts to understand this problem are hindered by the subtle interplay among a few mechanisms and the presence of several nearly degenerate and competing phases in these systems. Here we present some crucial experiments that place essential constraints on the pairing mechanism of high-temperature superconductivity. The observed unconventional oxygenisotope effects in cuprates have clearly shown strong electron-phonon interactions and the existence of polarons and/or bipolarons. Angle-resolved photoemission and tunneling spectra have provided direct evidence for strong coupling to multiple-phonon modes. In contrast, these spectra do not show strong coupling features expected for magnetic resonance modes. Angle-resolved photoemission spectra and the oxygen-isotope effect on the antiferromagnetic exchange energy $J$ in undoped parent compounds consistently show that the polaron binding energy is about $2 \mathrm{eV}$, which is over one order of magnitude larger than $J=0.14 \mathrm{eV}$. The normal-state spin-susceptibility data of holedoped cuprates indicate that intersite bipolarons are the dominant charge carriers in the underdoped region while the component of Fermi-liquid-like polarons is dominant in the overdoped region. All the experiments to test the gap or order-parameter symmetry consistently demonstrate that the intrinsic gap (pairing) symmetry for the Fermi-liquid-like component is anisotropic $s$-wave and the order-parameter symmetry of the Bose-Einstein condensation of bipolarons is $d$-wave.
\end{abstract}

\section{BCS THEORY AND THE CONVENTIONAL ISOTOPE EFFECT ON $T_{c}$}

In 1911 curiosity concerning the electrical properties of metals at low temperatures led the Dutch physicist, $\mathrm{H}$. K. Onnes and his assistant G. Holst to discover superconductivity at $4.2 \mathrm{~K}$ in mercury [1]. This discovery was one of the most important experimental findings in low temperature physics. Since then tremendous theoretical and experimental efforts have been made with the aim of clarifying the microscopic mechanism responsible for this novel ground state.

On the long way towards a microscopic understanding of superconductivity, the observation of an isotope effect on $T_{c}$ in 1950 [2, 3] gave important clues to the understanding of the microscopic theory of superconductivity. The presence of an isotope effect thus implies that superconductivity is not of purely electronic origin. In the same year H. Fröhlich [4] pointed out that the electronphonon interaction gave rise to an indirect attractive interaction between electrons, which might be responsible for superconductivity. Fröhlich's theory played a decisive role in establishing the correct mechanism. Later, in 1956 L. Cooper [5] discovered that electrons with an attractive interaction form bound pairs (so called Cooper pairs) which lead to superconductivity. However, the existence of electron pairs does not necessarily imply a phonon mediated pairing. Indeed, Bose condensation as considered in 1955 by Schafroth [6], is also a possible mechanism for superconductivity, but the model was not able to explain the existence of an isotope effect. Finally, in 1957, Bardeen, Cooper and Schrieffer 7] developed the BCS theory that was the first successful microscopic theory of superconductivity.
It is remarkable that the BCS theory shows that

$$
k_{B} T_{c}=1.13 \hbar \omega \exp \left(-\frac{1}{N(0) V}\right),
$$

where $\omega$ is a typical phonon frequency (e.g., the Debye frequency $\left.\omega_{D}\right)$. The electron-phonon coupling constant $N(0) V$ is the product of an electron-phonon interaction constant $V$ and the electronic density of states at the Fermi surface $N(0)$, both of which are assumed to be independent of the ion mass $M$.

This formula implies an isotope-mass dependence of $T_{c}$, with an isotope-effect exponent $\alpha=-d \ln T_{c} / d \ln M$ $=1 / 2$, in excellent agreement with the reported isotope effects in the non-transition metal superconductors (e.g., $\mathrm{Hg}$, Sn and $\mathrm{Pb}$ ). On the other hand, many superconducting transition metals show a much smaller isotope effect (e.g. Zr, Ru: $\alpha=0 \pm 0.05$ ), and in some materials even negative isotope shifts have been reported (e.g. $\mathrm{U}: \alpha=-2.2 \pm 0.2$ ). The obvious discrepancy between the observed isotope effect and the prediction of the BCS theory demonstrates the limitations of the simplified BCS approach. Nevertheless, the phonon mediated BCS theory was the basis for further and more sophisticated microscopic theories of superconductivity.

Since the original BCS theory of 1957 there has been considerable progress toward a deeper understanding of the electron-phonon interaction. In the strong-coupling Eliashberg model [8] the electron-phonon coupling constant $\lambda_{e p}$ is related to the spectral function $\alpha^{2}(\omega) F(\omega)$, as defined by the product of the frequency-dependent average electron-phonon interaction $\alpha^{2}(\omega)$ and phonon density of states $F(\omega)$. McMillan [9] numerically solved the Eliashberg equations and found an expression for $T_{c}$ taking into account the Coulomb repulsion between electrons 
and the retarded nature of the phonon-induced interaction

$$
k_{B} T_{c}=\frac{\hbar \omega_{D}}{1.45} \exp \left(-\frac{1.04\left(1+\lambda_{e p}\right)}{\lambda_{e p}-\mu^{*}\left(1+0.62 \lambda_{e p}\right)}\right),
$$

where $\mu^{*}$ is an effective Coulomb repulsion. The BCS result of Eq. (1) is recovered for $\lambda_{e p} \ll 1$, and in this weakcoupling limit $\lambda_{e p}-\mu^{*}$ substitutes the role of $N(0) V$. As a consequence, in strong-coupling superconductors the isotope effect exponent $\alpha$ is no longer a universal value and given by

$$
\alpha=\frac{1}{2}\left(1-\frac{1.04\left(1+0.62 \lambda_{e p}\right)\left(1+\lambda_{e p}\right) \mu^{* 2}}{\left[\lambda_{e p}-\mu^{*}\left(1+0.62 \lambda_{e p}\right)\right]^{2}}\right) .
$$

Within the framework of the Eliashberg theory a small or even negative isotope exponent is possible. Therefore, the observation of a small isotope exponent could still be consistent with the phonon-mediated pairing mechanism.

\section{ISOTOPE EFFECT ON $T_{c}$ IN HIGH- $T_{c}$ CUPRATE SUPERCONDUCTORS}

Since the discovery of superconductivity in $\mathrm{La}_{2-x} \mathrm{Ba}_{x} \mathrm{CuO}_{4}$ by Bednorz and Müller in 1986 [10], tremendous efforts have been made to clarify the microscopic pairing mechanism for high-temeprature superconductivity. In some of the cuprates the critical temperatures are far beyond a supposed maximum $T_{c} \approx 30 \mathrm{~K}$, estimated theoretically on the basis of the conventional phonon-mediated mechanism [9]. However, the theoretical estimate has not been well justified.

Contrary to low $T_{c}$ elemental superconductors, the cuprates have a complex crystal structure and consist of at least three constituents, leading to complicated phononic and electronic densities of states. The structure of cuprates may be described by the three main features: i) the $\mathrm{CuO}_{2}$ planes, ii) the apical oxygen, iii) the metal-oxygen charge reservoir. All the high- $T_{c}$ superconductors have $\mathrm{CuO}_{2}$ planes, but the number of planes varies among the different families of cuprates, ranging from a single-layer up to an infinite-layer structure.

The investigation of the isotope effect in cuprates is certainly much more difficult than in elemental superconductors. In compound superconductors, such as the cuprates, it is convenient to define a partial isotope exponent for a constituent with mass $M_{i}$ as

$$
\alpha_{i}=-\frac{\Delta T_{c}^{i}}{T_{c}} \frac{M_{i}}{\Delta M_{i}}
$$

where the index $i$ denotes a constituent with mass $M_{i}$ (e.g. oxygen). The magnitude of $\alpha_{i}$ may provide insight into which phonons are important in the occurrence of superconductivity. As discussed above, small or even vanishing partial isotope exponents do not necessarily imply that the corresponding constituent is not important for the occurrence of superconductivity. For a conclusive decision on the role of lattice vibrations in superconductivity, one should not only study the isotope effect on $T_{c}$, but also the isotope effects on some other parameters such as the effective supercarrier mass $m^{*}$ and the supercarrier density $n_{s}$. The latter effects may offer more information about the role of phonons, and will be crucial to the understanding of the physics of high-temperature superconductors.

Studies of isotope shifts of $T_{c}$ have been carried out in almost all known cuprates. A comprehensive review is given by Franck 11]. Most of the studies reported so far concern the oxygen isotope shift by replacing ${ }^{16} \mathrm{O}$ with ${ }^{18} \mathrm{O}$, partly because the experimental procedures are simple and reliable. Today it is generally accepted that the cuprates doped for maximum $T_{c}$ exhibit a small but clearly non-vanishing $\alpha_{O}$. This is true for all the different families of doped cuprates. Some of the results are listed in Table. []

\begin{tabular}{lcc}
\hline \hline & $T_{c}(\mathrm{~K})$ & \multicolumn{2}{c}{ Oxygen-isotope exponent $\alpha_{O}$} \\
\hline & & \\
$\mathrm{La}_{1.85} \mathrm{Sr}_{0.15} \mathrm{CuO}_{4}$ & $35-37$ & $0.08-0.13$ \\
$\mathrm{YBa}_{2} \mathrm{Cu}_{3} \mathrm{O}_{6.94}$ & $90-92$ & $0.02-0.03$ \\
$\mathrm{YBa}_{2} \mathrm{Cu}_{4} \mathrm{O}_{8}$ & 79.5 & $0.06-0.08$ \\
$\mathrm{Bi}_{2} \mathrm{Sr}_{2} \mathrm{CaCu}_{2} \mathrm{O}_{8+y}$ & 75 & $0.03-0.05$ \\
$\mathrm{Tl}_{2} \mathrm{Ca}_{2} \mathrm{Ba}_{1} \mathrm{Cu}_{3} \mathrm{O}_{10}$ & 121 & $0 \pm 0.12$ \\
$\mathrm{Nd}_{1.85} \mathrm{Ce}_{0.15} \mathrm{CuO}_{4}$ & 24.5 & $<0.05$ \\
& & \\
\hline \hline
\end{tabular}

TABLE I: Oxygen isotope effect for various cuprates doped for optimum $T_{c}$ (compiled from Ref. [11]) .

Although the different compounds exhibit quite different $T_{c}$ 's, it is striking that the oxygen-isotope exponents (OIEs) are similar in magnitude and remain small. This suggests that the OIE depends neither on $T_{c}$ nor on specific structural properties such as the number of $\mathrm{CuO}_{2}$ planes and the electronic properties of the metal-oxygen buffer layers. The small OIE observed in the optimally doped cuprates suggest that phonons might not be important in high temperature superconductivity. This has led to many exotic non-phonon mediated pairing mechanisms (e.g., see Ref. [12]).

However, the doping dependence of the OIE has been extensively studied in different cuprate systems 13 19. For a particular family of doped cuprates the OIE increases with decreasing $T_{c}$, and can be even larger than the BCS prediction 13]. Such an anomalously large OIE may imply that phonons play an important role in the occurrence of high temperature superconductivity in the copper oxides. Any correct theories for describing the physics of high- $T_{c}$ superconductors must consistently explain both the small OIE in the optimally-doped samples, and the anomalously large OIE in the underdoped samples. 
The non-vanishing OIEs indicate that the oxygendominated phonon modes are involved in the occurrence of superconductivity in the cuprates. As we will see below, there are strong coupling features to multiple phonon modes in tunneling and angle-resolved photoemission spectra. This implies that there should be isotope shifts related to other atoms. Indeed, large copperisotope shifts, with $\alpha_{C u}$ being close to $\alpha_{O}$, have been observed in underdoped $\mathrm{La}_{2-x} \mathrm{Sr}_{x} \mathrm{CuO}_{4}$ (LSCO) [15] and in oxygen-depleted $\mathrm{YBa}_{2} \mathrm{Cu}_{3} \mathrm{O}_{7-y}[20]$. This implies that $\mathrm{Cu}$-dominated phonon modes also play important role in the pairing.

\section{OXYGEN-ISOTOPE EFFECT ON THE IN-PLANE SUPERCARRIER MASS IN CUPRATES}

The conventional phonon-mediated superconducting theory is based on the Migdal adiabatic approximation in which the phonon-induced electron self-energy is given correctly to order $(m / M)^{1 / 2} \sim 10^{-2}$, where $m$ is the mass of an electron. Within this approximation, the density of states at the Fermi level $N(0)$, the electronphonon coupling constant $\lambda_{e p}$, and the effective mass of the supercarriers are all independent of the ion-mass $M$. This approximation has been working very well in conventional elemental superconductors because the ratio of the sound velocity is much smaller than the Fermi velocity. However, since the phase velocity of long wavelength optical phonons is large compared to the Fermi velocity, it may break down in some compounds with reasonably strong electron-phonon interactions 21]. The break-down of the Migdal approximation may lead to the formation of polarons-new quasi-particles moved together with local lattice distortions. The effective mass of polarons $m^{*}$ will depend on $M$. This is because the polaron mass $m^{*}=m \exp \left(\gamma E_{p} / \hbar \omega\right)$ 22, 23], where $m$ is the band mass in the absence of the electron-phonon interaction, $\gamma$ is a constant, and $\omega$ is a characteristic phonon frequency which depends on the masses of ions. Hence, there should be a large isotope effect on the polaronic carrier mass, in contrast to the zero isotope effect on the effective carrier mass in ordinary metals. Due to the polaronic band narrow effect, the effective density of states at the Fermi level $N(0)$ and the effective electron-phonon coupling constant $\lambda_{e p}$ [proportional to $N(0)$ ] should also depend on the ion mass $M$. This will lead to more complicated isotope effect on $T_{c}$. Therefore, negative, vanishing, or small isotope exponents $\alpha \ll 1 / 2$ in optimally doped cuprates do not necessarily imply that phonons are not important for the pairing mechanism if polarons are bound into the Cooper pairs.

The total exponent of the isotope effect on the effective carrier mass is defined as $\beta=\sum-d \ln m^{*} / d \ln M_{i}\left(M_{i}\right.$ is the mass of the $i$ th atom in a unit cell). For polaronic carriers $m^{*}=m \exp \left(\gamma E_{p} / \hbar \omega\right)$, this definition leads to

$$
\beta=-\frac{1}{2} \ln \left(m^{*} / m\right)
$$

It is interesting that the simple relation $m^{*}=$ $m \exp \left(\gamma E_{p} / \hbar \omega\right)$ is even valid in the weak coupling region in the case of the long-range Fröhlich electron-phonon interaction [23]. Then the polaron mass enhancement factor $m^{*} / m$ in this case is simply equal to $\exp (-2 \beta)$. Therefore, if electron-phonon coupling in a solid is strong enough to form polarons and/or bipolarons, one should expect a substantial isotope effect on the effective mass of carriers.

An important and essential proof for the existence of polarons and/or bipolarons in cuprates is provided by the observation of the substantial oxygen-isotope effect on the penetration depth [16, 19, 24 31]. Zhao et al. made the first observation of this effect in optimally doped $\mathrm{YBa}_{2} \mathrm{Cu}_{3} \mathrm{O}_{6.93}$ in 1995 [24]. By precisely measuring the diamagnetic signals near $T_{c}$ for the ${ }^{16} \mathrm{O}$ and ${ }^{18} \mathrm{O}$ samples, the authors were able to deduce the oxygen-isotope effect on the penetration depth $\lambda(0)$. It turns out that $\Delta \lambda(0) / \lambda(0)=3.2 \%$ (Ref. [24]).

In fact, for highly anisotropic materials, the observed isotope effect on the angle-averaged $\lambda(0)$ is the same as the isotope effect on the in-plane penetration depth $\lambda_{a b}(0)$. From the magnetic data for $\mathrm{YBa}_{2} \mathrm{Cu}_{3} \mathrm{O}_{6.93}$, $\mathrm{La}_{1.85} \mathrm{Sr}_{0.15} \mathrm{CuO}_{4}$, and $\mathrm{Bi}_{1.6} \mathrm{~Pb}_{0.4} \mathrm{Sr}_{2} \mathrm{Ca}_{2} \mathrm{Cu}_{3} \mathrm{O}_{10+y}$, one finds that $\Delta \lambda_{a b}(0) / \lambda_{a b}(0)=3.2 \pm 0.7 \%$ for the three optimally doped cuprates [29]. Several independent experiments have consistently shown that the carrier densities of the two isotope samples are the same within 0.0004 per unit cell [19, 25, 27, 30]. Therefore, the observed oxygen-isotope effect on the in-plane penetration depth is caused only by the isotope dependence of the inplane supercarrier mass. Recently, direct measurements of the in-plane penetration depth by low energy muonspin-relaxation (LE $\mu \mathrm{SR}$ ) technique [30] have confirmed the earlier isotope-effect results. It is found that 30] $\Delta \lambda_{a b}(0) / \lambda_{a b}(0)=2.8 \pm 1.0 \%$. It is remarkable that the isotope effect obtained from the most advanced and expensive technology $(\mathrm{LE} \mu \mathrm{SR})$ is the same as that deduced from simple magnetic measurements.

Fig. 1 shows the doping dependence of the exponent $\left(\beta_{O}\right)$ of the oxygen-isotope effect on the in-plane supercarrier mass in $\mathrm{La}_{2-x} \mathrm{Sr}_{x} \mathrm{CuO}_{4}$. Here the exponent is defined as $\beta_{O}=-d \ln m_{a b}^{* *} / d \ln M_{O}$. It is apparent that the exponent increases with decreasing doping, in agreement with the fact that doping reduces electron-phonon coupling due to screening of charge carriers. The large oxygen-isotope effect on the in-plane supercarrier mass cannot be explained within the conventional phononmediated pairing mechanism where the effective mass of supercarriers is independent of the isotope mass [32]. In particular, the substantial oxygen-isotope effect on $m_{a b}^{* *}$ in optimally doped cuprates indicates that the polaronic effect is not vanished in the optimal doping regime where 


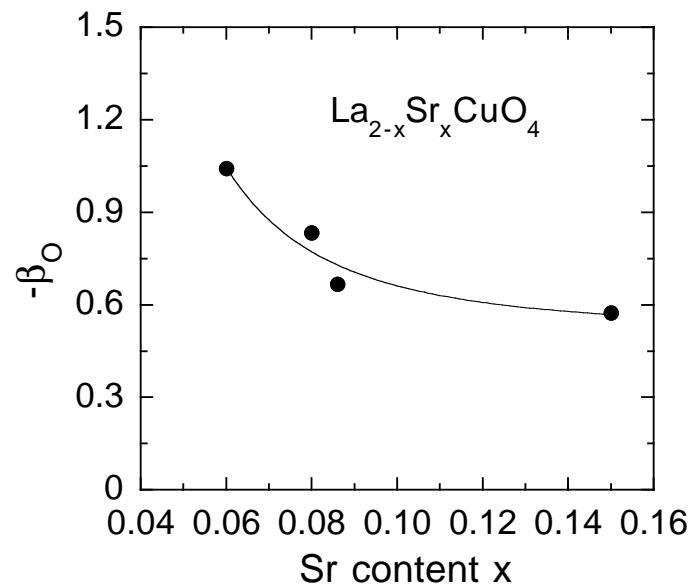

FIG. 1: The doping dependence of the exponent $\beta_{O}$ of the oxygen-isotope effect on the in-plane supercarrier mass in $\mathrm{La}_{2-x} \mathrm{Sr}_{x} \mathrm{CuO}_{4}$. The exponent is defined as $\beta_{O}=$ $-d \ln m_{a b}^{* *} / d \ln M_{O}$. The data are from Ref. [26, 28, 29]

the BCS-like superconducting transition occurs. This suggests that polaronic carriers may be bound into the Cooper pairs in optimally doped and overdoped cuprates. This scenario [22, 29] can naturally explain a small isotope effect on $T_{c}$ in optimally doped cuprates.

\section{DETERMINATION OF THE ELECTRON-PHONON COUPLING STRENGTH IN UNDOPED PARENT CUPRATES}

The observed substantial oxygen-isotope effect on the effective supercarrier mass suggests that doped holes are strongly coupled with phonons to form polarons and/or bipolarons. It is natural that undoped parent compounds like $\mathrm{La}_{2} \mathrm{CuO}_{4}$ should have stronger electron-phonon interactions because no doped charge carriers can screen the electron-phonon interactions. Very strong electronphonon interactions can reduce electron hopping integral $t$ through the polaronic effect [22], which may lead to the renormalization of magnetic exchange energy. Then one might expect that the antiferromagnetic (AF) exchange energy in antiferromagnetic cuprates and the ferromagnetic exchange energy in ferromagnetic manganites should depend on the isotope mass if both systems have very strong electron-phonon coupling. Following this simple argument, Zhao and his co-workers initiated studies of the oxygen-isotope effect on the AF ordering temperature $T_{N}$ in several parent cuprates [33] and the oxygen-isotope effect on the Curie temperature in ferromagnetic managnites [34]. A small oxygen-isotope shift of $T_{N}$ was consistently observed in $\mathrm{La}_{2} \mathrm{CuO}_{4}$ [33] while a giant oxygen-isotope shift of the Curie temperature was observed in doped manganites [34]. The large difference in the isotope effects on the magnetic ordering temperatures appears contradictory since both materials should have similar electron-phonon coupling strengths. Here we will show that the small isotope shift of $T_{N}$ is also consistent with a large polaron binding energy due to the fact that the electron-phonon interaction renormalizes the antiferromagnetic exchange energy $J$ through the forth order process [35].

From the oxygen-isotope shift of $T_{N}$, one can determine the oxygen-isotope effect on the antiferromagnetic exchange energy $J$, that is, $\Delta J / J \simeq-0.6 \%$ (Ref. 33]). A slightly larger oxygen-isotope effect on $J(\Delta J / J \simeq$ $-0.96 \%)$ has also been extracted from the mid-infrared two-magnon absorption spectra of the oxygen-isotope exchanged $\mathrm{YBa}_{2} \mathrm{Cu}_{3} \mathrm{O}_{6.0}$ (Ref. [36]). This unconventional isotope effect on $J$ can be explained in terms of strong electron-phonon coupling and polaronic effect. Eremin et al. 35] have considered strong electron-phonon coupling within a three-band Hubbard model appropriate for the charge-transfer insulators. They show that the antiferromagnetic exchange energy $J$ depends on the polaron binding energy $E_{p}^{O}$ due to oxygen vibrations, on the polaron binding energy $E_{p}^{C u}$ due to copper vibrations, and on their respective vibration frequencies $\omega_{O}$ and $\omega_{C u}$. At low temperatures, $J$ is given by 35]

$$
J=J_{\circ}\left(1+\frac{3 E_{p}^{O} \hbar \omega_{O}}{\Delta_{p d}^{2}}+\frac{3 E_{p}^{C u} \hbar \omega_{C u}}{\Delta_{p d}^{2}}\right) .
$$

Here $\Delta_{p d}$ is the charge-transfer gap, which is measured to be about $1.5 \mathrm{eV}$ in undoped cuprates. From the above equation, one can clearly see that there should be an observable oxygen-isotope effect on $J$ if the polaron binding energy is significant. The oxygen-isotope effect on $J$ can be readily deduced from Eq. 6.

$$
\frac{\Delta J}{J}=\left(\frac{3 E_{p}^{O} \hbar \omega_{O}}{\Delta_{p d}^{2}}\right)\left(\frac{\Delta \omega_{O}}{\omega_{O}}\right) .
$$

The charge-transfer gaps $\Delta_{p d}$ have been measured for both $\mathrm{La}_{2} \mathrm{CuO}_{4}$ and $\mathrm{YBa}_{2} \mathrm{Cu}_{3} \mathrm{O}_{6}$ systems [37], that is, $\Delta_{p d}=1.81 \mathrm{eV}$ for $\mathrm{La}_{2} \mathrm{CuO}_{4}$ and $\Delta_{p d}=1.60 \mathrm{eV}$ for $\mathrm{YBa}_{2} \mathrm{Cu}_{3} \mathrm{O}_{6}$. If we take $\hbar \omega_{O}=0.075 \mathrm{eV}$ and substitute the above parameters into Eq. 7 we obtain $E_{p}^{O}=1.46 \mathrm{eV}$ for $\mathrm{La}_{2} \mathrm{CuO}_{4}$ and $E_{p}^{O}=1.82 \mathrm{eV}$ for $\mathrm{YBa}_{2} \mathrm{Cu}_{3} \mathrm{O}_{6}$. Since the copper-related phonon modes have much lower energies than the oxygen-related modes, the copper-related phonon modes may contribute little to the polaron formation, that is, $E_{p}^{C u}<<1$. Therefore, the total polaron binding energy $E_{p}$ should be about $1.5 \mathrm{eV}$ and $1.9 \mathrm{eV}$ for $\mathrm{La}_{2} \mathrm{CuO}_{4}$ and $\mathrm{YBa}_{2} \mathrm{Cu}_{3} \mathrm{O}_{6}$, respectively.

Angle-resolved photoemission spectroscopy (ARPES) data of undoped $\mathrm{La}_{2} \mathrm{CuO}_{4}$ have been explained in terms of polaronic coupling between phonons and charge carriers [38]. From the width of the phonon side band in the ARPES spectra, the authors find the polaron binding energy to be about $1.92 \mathrm{eV}$, in good agreement with their theoretical calculation based on a shell model [38]. On the other hand, the observed binding energy of the 
side band should be consistent with a polaron binding energy of about $1.2 \mathrm{eV}$ (Ref. 38]). This should be the lower limit because the binding energy of the side band decreases rapidly with doping and because the sample may be lightly doped [38]. Therefore, the ARPES data suggest that $1.2 \mathrm{eV}<E_{p}<1.9 \mathrm{eV}$, which is in quantitative agreement with the value $\left(E_{p}>1.5 \mathrm{eV}\right)$ deduced from the isotope effect on the exchange energy.

The parameter-free estimate of the polaron binding energy due to the long-range Fröhlich-type electron-phonon interaction has been made for many oxides including cuprates and manganites [39]. The polaron binding energy due to the long-range Fröhlich-type electron-phonon interaction is estimated to be about $0.65 \mathrm{eV}$ for $\mathrm{La}_{2} \mathrm{CuO}_{4}$ (Ref. 39]). The polaron binding energy due to the $Q_{1}$ type Jahn-Teller distortion is about $1.2 \mathrm{eV}$ for $\mathrm{La}_{2} \mathrm{CuO}_{4}$ (Ref. [40]). The total polaron binding energy $E_{p}$ should be at least $1.85 \mathrm{eV}$, in excellent agreement with the value $\left(1.5 \mathrm{eV}<E_{p}<1.9 \mathrm{eV}\right.$ ) deduced independently from the oxygen-isotope effect on $J$ and ARPES data.

If there are very small amounts of charged carriers in these nearly undoped compounds, the optical conductivity due to the polaronic charge carriers will show a broad peak at $E_{m}=2 \gamma E_{p}$ (Ref.[39]), where $\gamma$ is $0.2-0.3$ for layered cuprates [39]. There appears to exist the third broad peak at $0.7-0.8 \mathrm{eV}$ in the optical conductivity of $\mathrm{Sr}_{2} \mathrm{CuO}_{2} \mathrm{Cl}_{2}$ (Ref. [41]), $\mathrm{La}_{2} \mathrm{CuO}_{4}$ (Ref. [42]), and $\mathrm{YBa}_{2} \mathrm{Cu}_{3} \mathrm{O}_{6}$ (Ref. [43]). This peak should be caused by the polaronic effect because the energy scale for the peak is similar to that predicted from the polaron theory assuming $\gamma \sim 0.2$. Hole doping will reduce the value of $E_{p}$ and thus $E_{m}$ due to screening of charged carriers. Indeed, $E_{m}$ was found to be about $0.6 \mathrm{eV}$ for $\mathrm{La}_{1.98} \mathrm{Sr}_{0.02} \mathrm{CuO}_{4}$ and $0.44 \mathrm{eV}$ for $\mathrm{La}_{1.94} \mathrm{Sr}_{0.06} \mathrm{CuO}_{4}$ (Ref. [44]).

\section{STRONG COUPLING TO MULTIPLE-PHONON MODES IN ANGLE-RESOLVED PHOTOEMISSION SPECTROSCOPY AND TUNNELING SPECTRA}

It is known that the electron self-energy renormalization effect in the form of a "kink" in the band dispersion can reveal coupling of electrons with phonon modes. The "kink" feature at an energy of about $70 \mathrm{meV}$ has been seen in the band dispersion of various cuprate superconductors along the diagonal ("nodal") direction 45. The energy scale of about $70 \mathrm{meV}$ appears to provide evidence for strong coupling between electrons and the $70 \mathrm{meV}$ $\mathrm{Cu}-\mathrm{O}$ half-breathing mode observed by neutron scattering [46]. From the measured dispersion, one can extract the real part of electron self-energy that contains information about coupling of electrons with collective boson modes. The fine coupling structures can be revealed in the high-resolution ARPES data. Very recently, such fine coupling structures have been clearly seen in the raw data of electron self-energy of deeply underdoped
$\mathrm{La}_{2-x} \mathrm{Sr}_{x} \mathrm{CuO}_{4}$ along the diagonal direction [47]. Using the maximum entropy method (MEM) procedure, they are able to extract the electron-phonon spectral density $\alpha^{2} F(\omega)$ that contains coupling features at $27 \mathrm{meV}, 45$ $\mathrm{meV}, 61 \mathrm{meV}$ and $75 \mathrm{meV}$. These ARPES data and exclusive data analysis [47] clearly indicate that the phonons rather than the magnetic collective modes are responsible for the electron self-energy effect.

In fact, the fine coupling structures also appear in the earlier high-resolution ARPES data of a slightly overdoped BSCCO with $T_{c}=91 \mathrm{~K}$ (Ref. 48]). Zhao [49] reanalyzed the ARPES data and also showed strong coupling features at $21.4 \mathrm{meV}, 38.3 \mathrm{meV}, 44.4 \mathrm{meV}, 60.0 \mathrm{meV}$, and $76.2 \mathrm{meV}$, It is remarkable that the peak positions at $44.4 \mathrm{meV}, 60.0 \mathrm{meV}$, and $76.2 \mathrm{meV}$ for BSCCO match precisely with the peak positions at $45 \mathrm{meV}, 60 \mathrm{meV}$, and $76 \mathrm{meV}$ for underdoped $\mathrm{La}_{2-x} \mathrm{Sr}_{x} \mathrm{CuO}_{4}$ (Ref. [4]). This consistency suggests that the fine structures seen in the high-resolution ARPES data are indeed related to strong electron-phonon coupling to multiple phonon modes.

Strong electron-phonon coupling to multiple phonon modes have been also seen in several high-quality singleparticle tunneling spectra [50 53]. For conventional superconductors, strong electron-phonon coupling features clearly show up in single-particle tunneling spectra [32, [54, 55]. The energies of the phonon modes coupled to electrons can be precisely determined from tunneling spectra. More specifically, the energy positions of the peaks in $-\mathrm{d}^{2} I / \mathrm{d} V^{2}$, measured from the isotropic $s$-wave superconducting gap $\Delta$, correspond to those of the peaks in the electron-phonon spectral function $\alpha^{2}(\omega) F(\omega)$ (Refs. [32, 54, 55]). Therefore, if the coupling strength $\alpha^{2}(\omega)$ changes smoothly with $\omega$, the fine structures in the phonon density of states $F(\omega)$ will have a one-to-one correspondence to those in $-\mathrm{d}^{2} I / \mathrm{d} V^{2}$. Such a conventional approach to identify the electron-boson coupling features would have been extensively applied to high- $T_{c}$ superconductors if the superconducting gap were isotropic. Since the superconducting gap is highly anisotropic in high- $T_{c}$ superconductors, it is difficult to reliably determine the energies of bosonic modes if tunneling current is not directional. This may explain why the electron-boson coupling structures extracted from earlier tunneling spectra are not reproducible among different groups [50, 51, 56].

Zhao [52] analyzed high-quality tunneling spectra of $\mathrm{YBa}_{2} \mathrm{Cu}_{3} \mathrm{O}_{7-\delta}$ and $\mathrm{Bi}_{2} \mathrm{Sr}_{2} \mathrm{CaCu}_{2} \mathrm{O}_{8+\delta}$. The spectra of the second derivative of tunneling current $\mathrm{d}^{2} I / \mathrm{d} V^{2}$ in both compounds show clear dip and peak features due to strong coupling to the bosonic modes mediating electron pairing. The energy positions of nearly all the peaks in $-\mathrm{d}^{2} I / \mathrm{d} V^{2}$-like spectra match precisely with those in the phonon density of states obtained by inelastic neutron scattering. Similar results have also been reported in a $\mathrm{La}_{1.84} \mathrm{Sr}_{0.16} \mathrm{CuO}_{4}$ thin film [53]. These results consistently demonstrate that the bosonic modes mediating the electron pairing are phonons and that the large at- 
tractive interaction should arise primarily from strong coupling to multiple phonon modes.

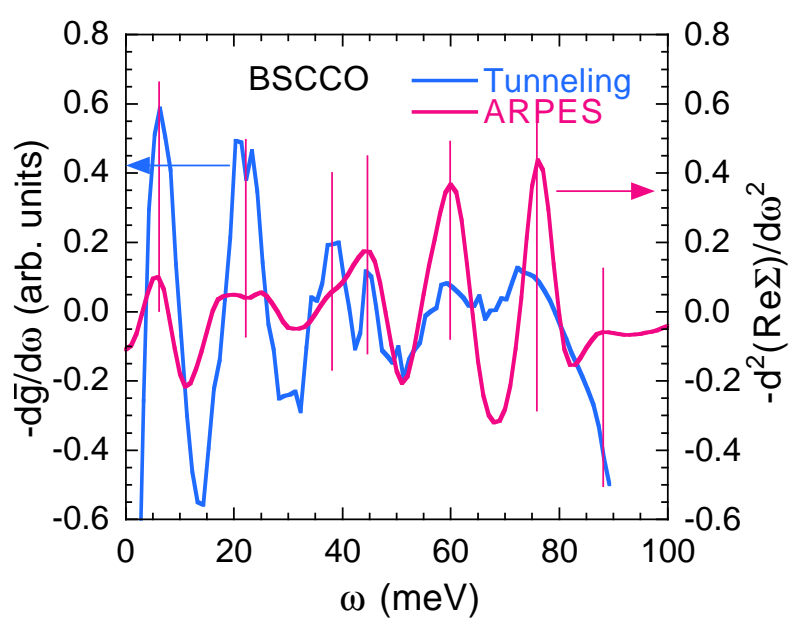

FIG. 2: The $-\mathrm{d}^{2}(\operatorname{Re} \Sigma) / \mathrm{d} \omega^{2}$ spectrum ( $\Sigma$ is electron selfenergy) and the $-\mathrm{d} \bar{g} / \mathrm{d} \omega$ tunneling spectrum of BSCCO crystal. After Ref. [49].

In Fig. 2, we compare the strong coupling features revealed in the tunneling spectrum and in the electron selfenergy spectrum of BSCCO. The spectrum of the second derivative of the real part of electron self-energy $(\Sigma)$ is reproduced from Ref. [49]. It is striking that the peak features in $\left(-\mathrm{d}^{2}(\operatorname{Re} \Sigma) / \mathrm{d} \omega^{2}\right)$ match very well with those in $(-\Delta(\mathrm{d} \bar{g} / \mathrm{d} \omega)$, where $\bar{g}$ is the renormalized tunneling conductance proportional to the first derivative of tunneling current [52]. This excellent agreement between the tunneling and self-energy spectra further demonstrates that the observed strong-coupling features in both spectra are intrinsic.

On the other hand, the authors of Ref. [57] mistakenly assigned the energy $(10.5 \mathrm{meV})$ of a peak position in $+\mathrm{d}^{2} I / \mathrm{d} V^{2}$ spectra of an electron-doped $\mathrm{Pr}_{0.88} \mathrm{LaCe}_{0.12} \mathrm{CuO}_{4}$ to the energy of a bosonic mode. Since this mistakenly assigned mode energy $(10.5 \mathrm{meV})$ is very close to the energy $(9.5-11 \mathrm{meV})$ of the magnetic resonance-like mode measured by inelastic neutron scattering [58, 59], the authors [57] conclude that the magnetic resonance mode mediates electron pairing in electron-doped cuprates. Zhao 60] reanalyzed the tunneling data and found strong coupling to a bosonic mode at about $16 \mathrm{meV}$, in quantitative agreement with early tunneling spectra of $\mathrm{Nd}_{1.85} \mathrm{Ce}_{0.15} \mathrm{CuO}_{4}$ (Ref. 61]). The correctly assigned mode energy of about $16 \mathrm{meV}$ rules out its connection to the magnetic resonance mode which has energy of $9.5 \mathrm{meV}$ in NCCO (Ref. [59]) and $11 \mathrm{meV}$ in PLCCO (Ref. [58]).

The pairing mechanism based on strong coupling to a magnetic resonance mode predicts a pronounced dip feature at the energy of the magnetic resonance mode in tunneling conductance spectra and angle-resolved photoemission spectra [62]. Some tunneling spectra of optimally doped BSCCO appear to support this prediction. For example, the energy of the dip feature in the tunneling spectrum of BSCCO is found to be about $45 \mathrm{meV}$ (Ref. [51]), close to the magnetic resonance energy of about $43 \mathrm{meV}$ (Ref. [63]). However, the inversion of the same tunneling spectrum [51] yields a very strong coupling feature at about $20 \mathrm{meV}$, in quantitative agreement with the mode energies of several overdoped BSCCO crystals inferred from the electron self-energies along the antinodal direction 64]. Furthermore, this theoretical prediction also contradicts more recent atomic-resolution tunneling spectra in various BSCCO crystals. The observed dip features in these crystals center around $26 \mathrm{meV}$ and are nearly independent of doping or $T_{c}[65]$. Since the energy of the magnetic resonance mode is found to be proportional to $T_{c}$ (Ref. [66]), the fact that the dip features are nearly independent of doping suggests that they are not caused by strong coupling to the magnetic resonance modes. Furthermore, the energy $(26 \mathrm{meV})$ of the dip feature in optimally doped BSCCO is too low compared with the energy $(43 \mathrm{meV}$ ) of the magnetic resonance mode and the energy $(35-40 \mathrm{meV})$ of the out-of-phase bond buckling oxygen modes 67].

Further evidence for no magnetic coupling is magnetooptical experiments on various cuprates [68, 69]. The data have shown that the electron-boson spectral function is independent of magnetic field [68], in contradiction with the theoretical prediction based on the magnetic pairing mechanism (see Fig. 9 of Ref. [68]).

\section{CO-EXISTENCE OF BIPOLARONS (FORMED BETWEEN APICAL AND IN-PLANE OXYGEN HOLES) WITH IN-PLANE OXYGEN HOLE POLARONS}

It is known that undoped parent cuprates are chargetransfer insulators, so doped holes should mainly reside on the oxygen orbitals. Bulk-sensitive x-ray-absorption experiment on $\left(\mathrm{Y}_{1-x} \mathrm{Ca}_{x}\right) \mathrm{Ba}_{2} \mathrm{Cu}_{3} \mathrm{O}_{7-y}$ confirms that doped holes mainly reside on both in-plane oxygen and apical oxygen orbitals 70]. What is striking is that doped holes via oxygen doping in the $\mathrm{CuO}$ chains are almost equally distributed to the apical and in-plane oxygen orbitals [70]. The Ca substitution for Y yields hole doping into the in-plane oxygen orbitals only and no superconductivity is seen in a compound with $10 \%$ in-plane oxygen holes and negligible apical oxygen holes. This remarkable experiment clearly demonstrates that the apical oxygen holes are crucial to high-temperature superconductivity. The data provide strong support for the theory of bipolaronic superconductivity proposed by Alexandrov and Mott 22]. The theory is based on mobile intersite bipolarons that are formed between apical and in-plane oxygen holes. Different types of bipolarons in $\mathrm{La}_{2} \mathrm{CuO}_{4}$ were investigated by Catlow et al. [71] with computer simulation techniques based on the minimization of the 
ground-state energy without the hopping term. An intersite bipolaron formed between an in-plane and an apical oxygen-hole polaron (denoted by AP bipolaron) was found energetically favorable with the binding energy of $0.14 \mathrm{eV}$. An intersite bipolaron formed between two inplane oxygen hole polarons (denoted by PP bipolaron) is also possible but with a much smaller binding energy $(0.06 \mathrm{eV})$. Both electron-phonon interactions and bipolaron binding energies will decrease when the hoping term is turned on. Doping will further reduce the bipolaron binding energy due to the screening of charge carriers. Therefore, it is possible that only AP bipolarons are stable in doped cuprates. For YBCO, about half of the inplane oxygen holes will be combined with the apical oxygen holes to form intersite bipolarons while the remaining in-plane oxygen holes are mixed with in-plane $\mathrm{Cu}$ holes and may be bound into $k$-space pairs below $T_{c}$. For LSCO, the number of the apical oxygen holes might be quite close to that of the in-plane oxygen holes since there are two apical and two in-plane oxygen atoms per formula unit.

An important proof for this simple model is provided by the data of the normal-state susceptibility $\chi(T)$ (Ref. 76, 77]). The temperature dependence of the normal-state susceptibility in cuprates is very different from conventional metals. It was found $[72$ that $\chi(T)$ exhibits a broad peak at a temperature $T_{\max }$. Such behavior was interpreted in terms of the 2-dimensional (2D) antiferromagnetic correlation among $\mathrm{Cu}$ spins. However, Nakano et al. [73] have shown that, at low temperatures, $\chi(T)$ strongly deviates from the prediction of the $2 \mathrm{D}$ AF model. Furthermore, the magnitude of $\chi(T)$ is proportional to the electronic specific heat [74], implying that the $\chi(T)$ reflects the density of states of the conduction electrons rather than the localized spin correlation. Alternatively, Alexandrov, Kabanov and Mott (AKM) explained the normal-state susceptibility data on the basis of their small (bi)polaron theory 75] which predicts a temperature-dependent spin susceptibility: $\chi_{A K M}(T)=B_{\infty} T^{-\frac{1}{2}} \exp \left(-\Delta_{b p} / 2 T\right)$, where $\Delta_{b p}$ is the bipolaron binding energy and $B_{\infty}$ is a constant depending on the effective masses of polarons and bipolarons [75]. If we consider possible coexistence with inplane oxygen-hole polaron Fermi-liquid, the total susceptibility for the two-component system [AP (bi)polarons + in-plane oxygen-hole polarons] is $\chi(T)=f_{s} \chi_{A K M}(T)$ $+\left(1-f_{s}\right) \chi_{F}+\chi_{c V}$. Here $f_{s}$ is the fraction of the AP (bi)polarons, $\chi_{F}$ is the susceptibility contributed from the Fermi-liquid-like polarons, and $\chi_{c V}$ is the total orbital contribution. Then

$$
\chi(T)=f_{s} B_{\infty} T^{-\frac{1}{2}} \exp \left(-\Delta_{b p} / 2 k_{B} T\right)+\chi_{\circ}
$$

Fig. 3 shows the temperature dependence of the normal-state susceptibility for $\mathrm{La}_{1-x} \mathrm{Sr}_{x} \mathrm{CuO}_{4}$. For $x=$ 0.20 , a small Curie paramagnetic susceptibility has been subtracted. The lowest solid line marks the total orbital contribution, as determined from the ${ }^{63} \mathrm{Cu}$ Knight shift

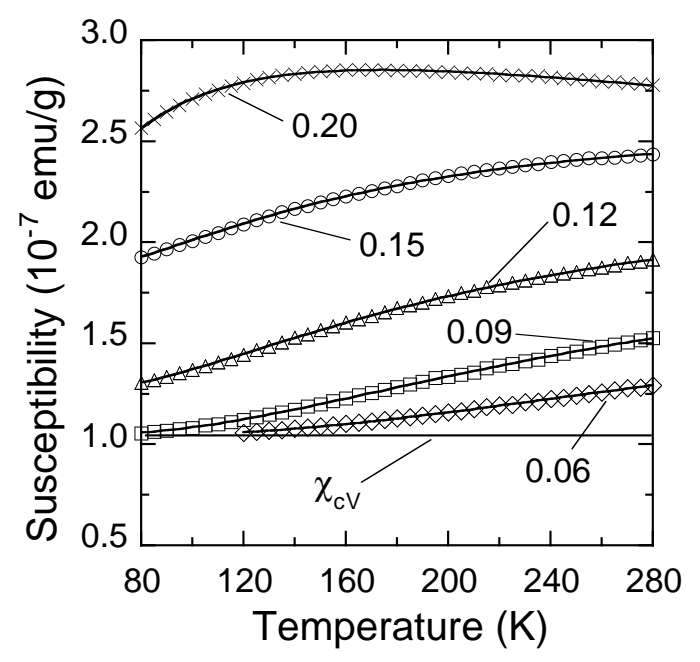

FIG. 3: The temperature dependence of the normal-state susceptibility for $\mathrm{La}_{1-x} \mathrm{Sr}_{x} \mathrm{CuO}_{4}$ (reproduced from Ref. 76]). The lowest solid line marks the total orbital contribution, as determined from the ${ }^{63} \mathrm{Cu}$ Knight shift of $\mathrm{La}_{1-x} \mathrm{Sr}_{x} \mathrm{CuO}_{4}$ (Ref. [78])

of $\mathrm{La}_{1-x} \mathrm{Sr}_{x} \mathrm{CuO}_{4}$ (Ref. [78]). The solid lines in Fig. 3 represent the fitting curves using Eq. 8. The fitting is excellent for all the compositions. It is interesting to see that for $x>0.09,\left(1-f_{s}\right) \chi_{F} \neq 0$, that is, the Fermiliquid type carriers are also present. From the more extended $\chi(T)$ data 74 , one sees that for $x \leq 0.10$, only (bi)polaronic charge carriers exist, and that the fraction of the Fermi-liquid carriers increases monotonically with $x$ for $x>0.10$. Therefore, the theory of bipolaronic superconductivity should be applied for $x \leq 0.10$. For $x>$ 0.1 , it is possible that, due to a decrease of the chargetransfer gap, more in-plane $\mathrm{Cu}$ holes are introduced so that the component of the Fermi-liquid-like carriers increases substantially. In the overdoped region (e.g., $x$ $=0.2$ ), the majority of the carriers are polarons at $T_{c}$, so superconductivity should arise from $k$-space pairing of Fermi-liquid-like polarons.

Now we discuss how this simple model is also compatible with ARPES results [79] for $\mathrm{La}_{1-x} \mathrm{Sr}_{x} \mathrm{CuO}_{4}$. The hole pocket centered around $(\pi / 2, \pi / 2)$ has been observed by ARPES for $x<0.18$. The hole pocket should be associated with the mixed states of the inplane oxygen $p$ orbitals and $\mathrm{Cu} d_{x^{2}-y^{2}}$ orbitals. A very flat band below the Fermi energy is seen near $(\pi, 0)$ at all the doping levels. This band has been attributed to the AP hole bipolaron state [80]. Above optimal doping, the hole pocket around $(\pi / 2, \pi / 2)$ appears to be connected with the hole pocket around $(\pi, 0)$ to form a large Fermi surface predicted by local density approximation. Nevertheless, the measured bare plasma energy for $x>0.15$ is only about 1.8 $\mathrm{eV}$ (Ref. [81]), which is much smaller than the predicted value of about $2.9 \mathrm{eV}$ (Ref. 82]). Therefore, the apparent connection between the two hole pockets may arise from the band-tailing phenomenon in doped semiconductors 
[83].

\section{THE INTRINSIC PAIRING SYMMETRY IN THE BULK OF CUPRATES}

An unambiguous determination of the pairing symmetry in cuprates is crucial to the understanding of the pairing mechanism of high-temperature superconductivity. Many experiments have been designed to test the pairing symmetry in the cuprate superconductors. However, contradictory conclusions have been drawn from different experimental techniques [84 90, 92 98, 119], which can be classified into being bulk-sensitive and surfacesensitive. For example, the magnetic penetration depth measurements and polarized Raman scattering experiments are bulk-sensitive. Angle-resolved photoemission spectroscopy is in general a surface-sensitive technique. However, the ARPES data for $\mathrm{Bi}_{2} \mathrm{Sr}_{2} \mathrm{CaCu}_{2} \mathrm{O}_{8+y}$ should nearly reflect the bulk properties since the cleaved top surface contains an inactive Bi-O layer, and the superconducting coherent length along the $c$-axis is very short. The single-particle tunneling experiments along the $\mathrm{CuO}_{2}$ planes can probe the bulk electronic density of states since the mean free path is far larger than the thickness of the degraded surface layer 99]. In contrast, the phase-sensitive experiments based on the planar Josephson tunneling are rather surface sensitive, so that they might not probe the intrinsic bulk superconducting state if the surfaces are strongly degraded. Therefore, the surface- and phase-sensitive experiments do not necessarily provide an acid test for the intrinsic bulk gap symmetry.

Zhao 100] has identified the intrinsic bulk pairing symmetry for hole-doped cuprates from the existing bulkand nearly bulk-sensitive experimental results such as ARPES, magnetic penetration depth, single-particle tunneling spectra, and nonlinear Meissner effect. These experimental results consistently show that the dominant pairing symmetry in hole-doped cuprates is an extended s-wave with eight line nodes, that is $\Delta(\theta)=$ $\Delta_{s}+\Delta_{g} \cos 4 \theta$, where the $g$ component $\Delta_{g}$ is larger than the $s$-wave component $\Delta_{s}$, and $\theta$ is measured from the $\mathrm{Cu}-\mathrm{O}$ bonding direction. Zhao [101] has also studied the intrinsic pairing symmetry for optimally electrondoped cuprates from bulk-sensitive data of Raman scattering, optical conductivity, magnetic penetration depth, directional point-contact tunneling spectra, and nonmagnetic pair-breaking effect. He has shown that all these bulk-sensitive data are in quantitative agreement with a nearly isotropic $s$-wave gap. The bulk-sensitive specific heat data of optimally electron-doped cuprates are also in quantitative agreement with a nearly isotropic $s$-wave gap [102].

In particular, bulk and phase-sensitive nonmagnetic pair-breaking effects can make clear distinction among different gap symmetries. Numerical calculations [101]

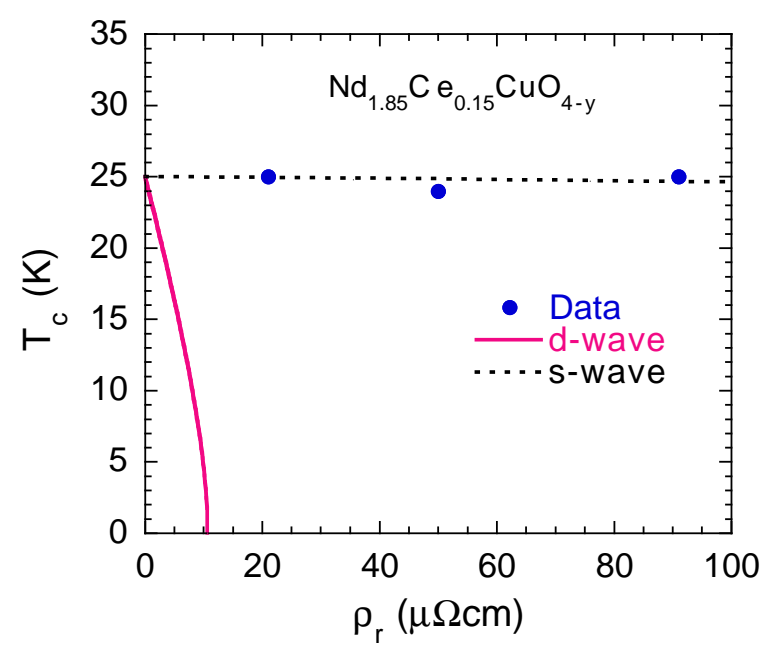

FIG. 4: $T_{c}$ as a function of residual resistivity in optimally doped $\mathrm{Nd}_{1.85} \mathrm{Ce}_{0.15} \mathrm{CuO}_{4-y}$. The solid line is numerically calculated curve in terms of any $d$-wave gap and the dotted line is the calculated curve for an $s$-wave gap function proportional to $(1-0.11 \cos 4 \theta)$. After Ref. [101].

for impurity pair-breaking effects indicate that $T_{c}$ can be suppressed to zero very rapidly with both magnetic and nonmagnetic impurities for a $d$-wave gap: $\Delta(\theta)=$ $\Delta_{d} \cos 2 \theta$ or a $g$-wave gap. In contrast, $T_{c}$ is hardly suppressed with nonmagnetic impurities for nearly isotropic $s$-wave gap 101]. The fact that $T_{c}$ is nearly independent of the residual resistivity in optimally electron-doped $\mathrm{Nd}_{1.85} \mathrm{Ce}_{0.15} \mathrm{CuO}_{4-y}$ (see Fig. 4) should rule out any $d$ wave gap including a nodeless $d$-wave gap [103].

Recent calculation based on $t-J$ model [104] shows that the nonmagnetic pair-breaking effect becomes less effective due to strong electron-electron correlation. This would explain the insensitivity of $T_{c}$ to the residual resistivity in the optimally electrondoped $\mathrm{Nd}_{1.85} \mathrm{Ce}_{0.15} \mathrm{CuO}_{4-y}$. However, this cannot consistently explain the very rapid $T_{c}$ suppression with the residual resistivity in a hole-doped $\mathrm{La}_{1.80} \mathrm{Sr}_{0.20} \mathrm{CuO}_{4}$ (Fig. 5b) unless the electron-electron correlation in $\mathrm{La}_{1.80} \mathrm{Sr}_{0.20} \mathrm{CuO}_{4}$ is negligibly small compared with that in $\mathrm{Nd}_{1.85} \mathrm{Ce}_{0.15} \mathrm{CuO}_{4-y}$. Since the renormalized plasma energy $\hbar \Omega_{p}^{*}=1.00 \mathrm{eV}$ (see below) of $\mathrm{La}_{1.80} \mathrm{Sr}_{0.20} \mathrm{CuO}_{4}$ is even much lower than that (1.64 eV) 105] for $\mathrm{Nd}_{1.85} \mathrm{Ce}_{0.15} \mathrm{CuO}_{4-y}$, the correlation effect of the former should be much stronger than that of the latter. If the $t-J$ model were relevant, $T_{c}$ of $\mathrm{Nd}_{1.85} \mathrm{Ce}_{0.15} \mathrm{CuO}_{4-y}$ would be suppressed more rapidly than that of $\mathrm{La}_{1.80} \mathrm{Sr}_{0.20} \mathrm{CuO}_{4}$. This is in total contradiction with the experimental results (see Figs. 4 and 5).

In fact, we can quantitatively explain the magnetic penetration depth and non-magnetic pair breaking effect in $\mathrm{La}_{1.80} \mathrm{Sr}_{0.20} \mathrm{CuO}_{4}$ even without taking into account electron-electron correlation. Fig. 5a shows the temperature dependence of the superfluid density $\left[\propto 1 / \lambda_{a b}^{2}(T)\right]$ for $\mathrm{La}_{1.80} \mathrm{Sr}_{0.20} \mathrm{CuO}_{4}$. The solid line is the numerically calculated curve for an extended $s$-wave $\left(s^{*}\right.$-wave) gap 

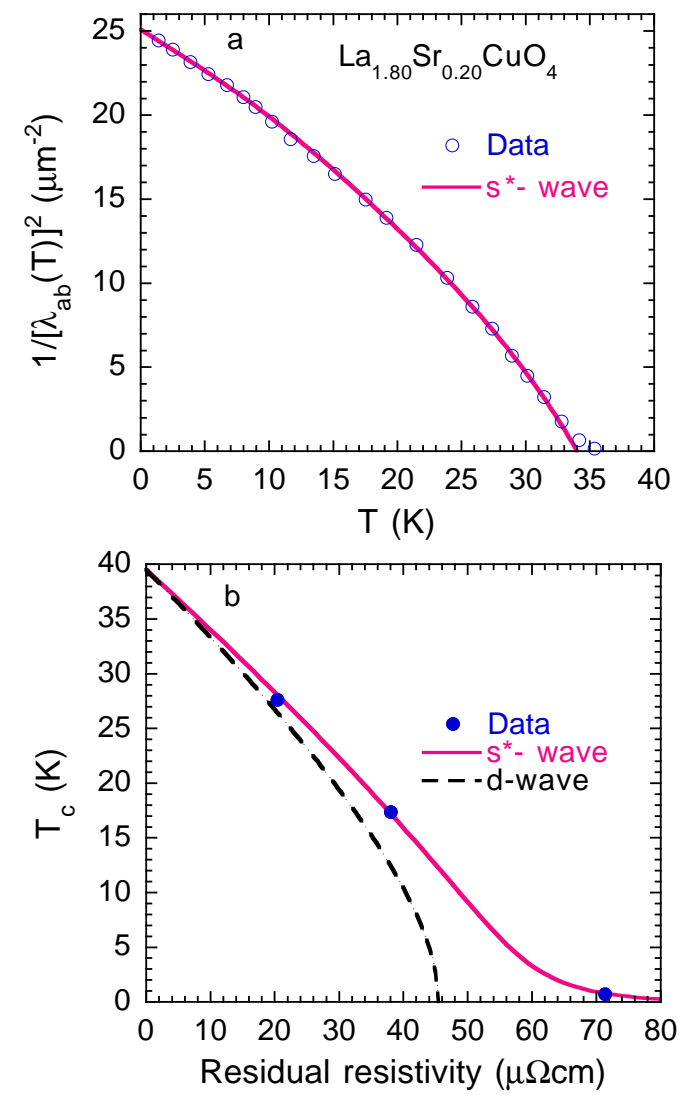

FIG. 5: a) Temperature dependence of the superfluid density $\left[\propto 1 / \lambda_{a b}^{2}(T)\right]$ for $\mathrm{La}_{1.80} \mathrm{Sr}_{0.20} \mathrm{CuO}_{4}$. The solid line is the numerically calculated curve for an extended $s$-wave gap function: $\Delta(\theta)=6.38(0.24+\cos 4 \theta) \mathrm{meV}$. The data are taken from Ref. [106]. b) $T_{c}$ dependence on the residual resistivity for $\mathrm{La}_{1.80} \mathrm{Sr}_{0.20} \mathrm{CuO}_{4}$. The solid line is calculated curve for the extended $s$-wave gap function: $\Delta(\theta)=6.38(0.24+\cos 4 \theta)$ $\mathrm{meV}$. The data are taken from Ref. [107].

function: $\Delta(\theta)=6.38(0.24+\cos 4 \theta) \mathrm{meV}$ using the standard formula 100 and the BCS temperature dependence of the gap. This gap function has a similar form as that for $\mathrm{YBa}_{2} \mathrm{Cu}_{3} \mathrm{O}_{7}$ with a similar doping [100]. Fig. 5b shows $T_{c}$ dependence on the residual resistivity for $\mathrm{La}_{1.80} \mathrm{Sr}_{0.20} \mathrm{CuO}_{4}$. The solid line is calculated curve for the extended $s$-wave gap function: $\Delta(\theta)=6.38(0.24+\cos 4 \theta) \mathrm{meV}$ obtained from the penetration depth data (Fig. 5a) using the standard formula [100] and the renormalized plasma energy $\hbar \Omega_{p}^{*}=1.00 \mathrm{eV}$ that is calculated from the measured zero-temperature penetration depth $(2000 \AA$ ) (see Fig. 5a). It is apparent that the calculated curve is in quantitative agreement with the data. In contrast, the calculated curve (dashed line) for $d$-wave gap is significantly off from the data.

When hole doping is reduced, the $s$-wave component increases so that the nonmagnetic pair-breaking effect becomes weaker. Since the bipolaronic charge carriers become dominant in underdoped cuprates, nonmagnetic impurities do not break the pairs, but can still suppress $T_{c}$ via localization effect.

With the two-carrier scenario in hole-doped cuprates, we can consistently explain surface and phase-sensitive experiments which probe $d$-wave order-parameter symmetry at surfaces and interfaces. Experiments on holedoped cuprates [108, 109] have shown that surfaces and interfaces are significantly underdoped so that bipolaronic charge carriers are dominant over polaronic Fermiliquid-like ones. Since the order-parameter symmetry of Bose-Einstein condensate of bipolarons are $d$-wave [110], the surface and phase-sensitive experiments should probe the dominant $d$-wave order-parameter symmetry, in agreement with experiments [92]. For electron-doped (n-type) cuprates, the penetration depth 111, 112] and point-contact tunneling spectra [113 117] consistently suggest that the gap symmetry in deeply underdoped samples should be $d$-wave and change to a nodeless $s$ wave when the doping level is above a critical value, in agreement with the theoretical prediction based on a phonon-mediated pairing mechanism [118]. This scenario can also explain the $d$-wave gap symmetry inferred from surface-sensitive experiments if surfaces or interfaces are deeply underdoped. Another possibility is that superconductivity in deeply underdoped $n$-type cuprates is also due to the Bose-Einstein condensation of local pairs. In this case, the superconducting quasi-particle gap is found to have a $d$-wave symmetry [119]. In sharp contrast to the standard theories of Bogoliubov quasi-particle excitations, the quasi-particle gap is shown [119] to originate from anomalous kinetic process, completely unrelated to the pairing symmetry.

\section{CONCLUDING REMARKS}

We have presented some crucial experiments that place essential constraints on the pairing mechanism of hightemperature superconductivity. The observed unconventional oxygen-isotope effects in cuprates have clearly shown strong electron-phonon interactions and the existence of polarons and/or bipolarons. Angle-resolved photoemission and tunneling spectra have provided direct evidence for strong coupling to multiple-phonon modes. In contrast, these spectra do not show strong coupling features expected for magnetic resonance modes. Angleresolved photoemission spectra and the oxygen-isotope effect on the antiferromagnetic exchange energy $J$ in undoped parent compounds consistently show that the polaron binding energy is about $2 \mathrm{eV}$, which is over one order of magnitude larger than $J=0.14 \mathrm{eV}$. In addition, a photoinduced lattice expansion experiment [120] and various optical data [121] also provide evidence for strong electron-phonon coupling.

The normal-state spin-susceptibility data of holedoped cuprates indicate that intersite bipolarons are the 
dominant charge carriers in the underdoped region while the component of Fermi-liquid-like polarons is dominant in the overdoped region. This two component scenario is also supported by the femtosecond optical spectroscopic experiment on $\left(\mathrm{Y}_{1-x} \mathrm{Ca}_{x}\right) \mathrm{Ba}_{2} \mathrm{Cu}_{3} \mathrm{O}_{7-y}$. Various bulksensitive experiments consistently demonstrate that the intrinsic gap (pairing) symmetry for the Fermi-liquidlike component is anisotropic $s$-wave while surface- and phase-sensitive experiments see $d$-wave order-parameter symmetry at intrinsically underdoped surfaces and interfaces where the dominant bipolaronic charge carriers are condensed into a $d$-wave superconducting state [110, 119].

* gzhao2@calstatela.edu

[1] H. Kammerling-Onnes, Comm. Physical Lab. Univ. Leiden, No. $120 \mathrm{~b}$ and $122 \mathrm{~b}$ (1911).

[2] E. Maxwell, Phys. Rev. 78, 477 (1950).

[3] C. A. Reynolds, B. Serin, W. H. Wright, and L. B. Nesbitt, Phys . Rev. 78, 487 (1950).

[4] H. Fröhlich, Phys. Rev. 79, 845 (1950).

[5] L. N. Cooper, Phys. Rev. 104, 1189 (1950).

[6] M. R. Schafroth, Phys. Rev. 100, 463 (1955).

[7] J. Bardeen, L. N. Cooper, and J. R. Schrieffer, Phys. Rev. 108, 1175 (1957).

[8] G. M. Eliashberg, Sov-Phys.-JETP 11, 696 (1960).

[9] W. L. McMillan, Phys. Rev. 167, 331 (1968).

[10] J. G. Bednorz and K. A. Müller, Z. Phys. B 64, 189 (1986).

[11] J. P. Franck, in: Physical Properties of High Temperature Superconductors IV (World Scientific, Singapore 1994), ed. by D. M. Ginsberg, p.189.

[12] P.W. Anderson, The Theory of Superconductivity in the High- $T_{c}$ Cuprate Superconductors, Princeton University Press, 1998.

[13] M. K. Crawford, M. N. Kunchur, W. E. Farneth, E. M. McCaron III, and S. J. Poon, Phys. Rev. B 41, 282(1990).

[14] H. J. Bornemann, D. E. Morris, H. B. Liu, and P. K. Narwankar, Physica C 191, 211 (1992).

[15] J. P. Franck, S. Harker, and J. H. Brewer, Phys. Rev. Lett. 71, 283 (1993).

[16] G. M. Zhao, K. K. Singh, A. P. B. Sinha, and D. E. Morris,, Phys. Rev. B 52, 6840 (1995).

[17] D. Zech, K. Conder, H. Keller, E. Kaldis, E. Liarokapis, N. Poulakis, and K. A. Müller, in: Anharmonic Properties of High- $T_{c}$ Cuprates, World Scientific, Singapore (1995). eds. D. Mihailović, G. Ruani, E. Kaldis, and K. A. Müller. p. 18.

[18] G. M. Zhao, J. W. Ager III, and D. E. Morris, Phys. Rev. B 54, 14982 (1996).

[19] G. M. Zhao, K. Conder, H. Keller, and K. A. Müller, J. Phys.: Condens. Matter, 10, 9055 (1998).

[20] G. M. Zhao et al., Phys. Rev. B 54, 14956 (1996).

[21] S. Engelsberg, and J. R. Schrieffer, Phys. Rev. 131, 993 (1963).

[22] A. S. Alexandrov and N. F. Mott, Polarons and Bipolarons, 67-106 (World Scientific, Singapore, 1995).

[23] A. S. Alexandrov and P. E. Kornilovitch, Phys. Rev.
Lett. 82, 807 (1999).

[24] G. M. Zhao and D. E. Morris, Phys. Rev. B 51, 16487(R) (1995).

[25] G. M. Zhao, M. B. Hunt, H. Keller, and K. A. Müller, Nature (London) 385, 236 (1997).

[26] J. Hofer, K. Conder, T. Sasagawa, G. M. Zhao, M. Willemin, H. Keller, and K. Kishio, Phys. Rev. Lett. 84, 4192 (2000).

[27] G. M. Zhao, H. Keller, and K. Conder, J. Phys.: Condens. Matter, 13, R569 (2001).

[28] G. M. Zhao, Phil. Mag. B 81, 1335 (2001).

[29] G. M. Zhao, V. Kirtikar, and D. E. Morris, Phys. Rev. B 63, 220506R (2001).

[30] R. Khasanov, D.G. Eshchenko, H. Luetkens, E. Morenzoni, T. Prokscha, A. Suter, N. Garifianov, M. Mali, J. Roos, K. Conder, and H. Keller, Phys. Rev. Lett. 92, 057602 (2004).

[31] R. Khasanov, A. Shengelaya, E. Morenzoni, K. Conder, I. M. Savic, and H. Keller, J. Phys.: Condens. Matter 16, S4439 (2004).

[32] J. P. Carbotte, Rev. Mod. Phys. 62, 1027 (1990).

[33] G. M. Zhao, K. K. Singh, and D. E. Morris, Phys. Rev. B 50, 4112 (1994).

[34] G. M. Zhao, K. Conder, H. Keller, K. A. Müller, Nature (London) 381, 676 (1996).

[35] I. Eremin, O. Kamaev, and M. V. Eremin, Phys. Rev. B 69, 094517 (2004).

[36] G. M. Zhao, Phys. Rev. B 75, 104511 (2007).

[37] S. L. Cooper, G. A. Thomas, A. J. Millis, P. E. Sulewski, J. Orenstein, D. H. Rapkine, S. W. Cheong, P. L. Trevor, Phs. Rev. B 42, 10785 (1990).

[38] O. Rösch, O. Gunnarsson, X. J. Zhou, T. Yoshida, T. Sasagawa, A. Fujimori, Z. Hussain, Z. X. Shen, and S. Uchida, Phys. Rev. Lett. 95227002 (2005).

[39] A S Alexandrov and A M Bratkovsky, J. Phys.: Condens. Matter 11, L531 (1999).

[40] H. Kamimura, Int. J. Mod. Phys. B 1, 873 (1987).

[41] H. S. Choi, E. J. Choi, and Y. J. Kim, Physica C 304, 66 (1998).

[42] J. D. Perkins, J. M. Graybeal, M. A. Kastner, R.J. Birgeneau, J. P. Falck, and M. Greven, Phys. Rev. Lett. 71, 1621 (1993).

[43] M. Gruninger, D. van der Marel, A. Damascelli, A. Erb, T. Nunner, and T. Kopp, Phys. Rev. B 62, 12422 (2000).

[44] X. X. Bi and P. C. Eklund, Phys. Rev. Lett. 702625 (1993).

[45] A. Lanzara, P. V. Bogdanov, X. J. Zhou, S. A. Kellar, D. L. Feng, E. D. Lu, T. Yoshida, H. Eisaki, A. Fujimori, K. Kishio, J.-I. Shimoyama, T. Nodak, S. Uchidak, Z. Hussain, and Z.-X. Shen, Nature (London) 412, 510 (2001).

[46] R. J. McQueeney, Y. Petrov, T. Egami, M. Yethiraj, G. Shirane, and Y. Endoh, Phys. Rev. Lett. 82, 628 (1999).

[47] X. J. Zhou et al., Phys. Rev. Lett. 95, 117001 (2005).

[48] P. D. Johnson, T. Valla, A.V. Fedorov, Z. Yusof, B. O. Wells, Q. Li, A. R. Moodenbaugh, G. D. Gu, N. Koshizuka, C. Kendziora, Sha Jian, and D. G. Hinks, Phys. Rev. Lett. 87, 177007 (2001).

[49] G. M. Zhao, Phys. Rev. B 75, 140510(R) (2007).

[50] D. Shimada, Y. Shiina, A. Mottate, Y. Ohyagi, and N. Tsuda, Phys. Rev. B 51, 16495 (1995).

[51] R. S. Gonnelli, G. A. Ummarino, and V. A. Stepanov, Physica C 275, 162 (1997).

[52] G. M. Zhao, Phys. Rev. B 75, 214507 (2007). 
[53] H. Shim, P. Chaudhari, G. Logvenov and I. Bozovic, Phys. Rev. Lett. 101, 247004 (2008).

[54] W. L. McMillan and J. M. Rowell, Phys. Rev. Lett. 14, 108 (1965).

[55] W. L. McMillan and J. M. Rowell, in Superconductivity, edited by R. D. Parks, (Marcel Dekker, New York, 1969), Vol. 1, p. 561; E. L. Wolf, Principles of electron tunneling spectroscopy, (Oxford University Press, New York, 1985); D. Shimada, N. Tsuda, U. Paltzer, F. W. de Wette, Physica C 298, 195 (1998).

[56] S. I. Vedeneev, P. Samuely, S. V. Meshkov, G. M. Eliashberg, A. G. M. Jansen, and P. Wyder, Physica C 198, 47 (1992).

[57] F. C. Niestemski, S. Kunwar, S. Zhou, Shiliang Li, H. Ding, Ziqiang Wang, Pengcheng Dai, and V. Madhavan,Nature (London) 450, 1058 (2007).

[58] Stephen D. Wilson1, Pengcheng Dai, Shiliang Li, Songxue Chi, H. J. Kang, and J. W. Lynn, Nature (London) 422, 59 (2006).

[59] Jun Zhao, Pengcheng Dai, Shiliang Li, Paul G. Freeman, Y. Onose, and Y. Tokura, Phys. Rev. Lett. 99, 017001 (2007).

[60] G. M. Zhao, Phys. Rev. Lett. 103, 236403 (2009).

[61] Q. Huang, J. F. Zasadzinski, N. Tralshawala, K. E. Gray, D. G. Hinks, J. L. Peng, and R. L. Greene, Nature (London) 347, 369 (1990).

[62] A. Abanov and A.V. Chubukov, Phys. Rev. Lett. 83, 1652 (1999).

[63] H. F. Fong, P. Bourges, Y. Sidis, L. P. Regnault, A. Ivanov, G. D. Guk, N. Koshizuka, and B. Keimer, Nature(London) 398, 588 (1999).

[64] G. M. Zhao, Phys. Rev. B 71, 104517 (2005).

[65] J.-H. Lee, K. Fujita, K. McElroy, J. A. Slezak, M. Wang, Y. Aiura, H. Bando, M. Ishikado, T. Masui, J.-X. Zhu, A. V. Balatsky, H. Eisaki, S. Uchida, and J. C. Davis, Nature 442, 546 (2006).

[66] H. He, Y. Sidis, P. Bourges, G. D. Gu, A. Ivanov, N. Koshizuka, B. Liang, C. T. Lin, L. P. Regnault, E. Schoenherr, and B. Keimer, Phys. Rev. Lett. 86, 1610 (2001).

[67] S. Johnston and T. P. Devereaux, Phys. Rev. B 81, $214512(2010)$.

[68] Y. S. Lee, K. Segawa, Z. Q. Li, W. J. Padilla, M. Dumm, S. V. Dordevic, C. C. Homes, Yoichi Ando, and D. N. Basov, Phys. Rev. B 72, 054529 (2005).

[69] S.V. Dordevic, L.W. Kohlman, L. C. Tung, Y.-J. Wang, A. Gozar, G. Logvenov, and I. Bozovic, Phys. Rev. B 79, 134503 (2009).

[70] M. Merz, N. Nücker, P. Schweiss, S. Schuppler, C. T. Chen, V. Chakarian, J. Freeland, Y. U. Idzerda, M. Kläser, G. Müller-Vogt, and Th. Wolf, Phys. Rev. Lett. 80, 5192 (1998).

[71] C. R. A. Catlow, M. S. Islam, and X. Zhang, J. Phys.: Condens. Matter 10, L49 (1998), and references therein.

[72] D. C. Johnston, Phys. Rev. Lett. 62, 957 (1989).

[73] T. Nakano et al., Phys. Rev. B49, 16000 (1994).

[74] J. W. Loram, K. A. Mirza, J. R. Cooper, N. Athanassopoulou, and W. Y. Liang, in Proceedings of the Aniversary HTS Workshop on Physics, Materials and Applications, edited by B. Batlogg, C. W. Chu, M. K. Chu, D. U. Gubser, and K. A. Müller (World Scientific, Singapore, 1996), p. 341.

[75] A. S. Alexandrov, V. V. Kabanov and N. F. Mott, Phys. Rev. Lett. 77, 4796 (1996).

[76] K A Müller, Guo-meng Zhao, K Conder, and H Keller,
J. Phys.: Condens. Matter, 10, L291 (1998).

[77] A. S. Alexandrov, V. N. Zavaritsky, and S. Dzhumanov, Phys. Rev. B 69, 052505 (2004)

[78] Y.-Q. Song et al., Phys. Rev. Lett. 70, 3131 (1993).

[79] A. Ino et al., Phys. Rev. B 62, 4137 (2000); T. Yoshida et al., Phys. Rev. Lett. 91, 027001 (2003).

[80] A. S. Alexandrov, Phys. Rev. B 53, 2863 (1996).

[81] K. Tamasaku, T. Ito, H. Takagi, and S. Uchida, Phys. Rev. Lett. 72, 3088 (1994).

[82] P. B. Allen, W. E. Pickett, and H. Krakauer, Phys. Rev. B 37, 7482 (1988).

[83] A. S. Alexandrov and K. Reynolds, Phys. Rev. B 76, 132506 (2007).

[84] W. N. Hardy, D. A. Bonn, D. C. Morgan, Ruixing Liang, and K. Zhang, Phys. Rev. Lett. 70, 3999 (1993).

[85] T. Jacobs, S. Sridhar, Q. Li, G. D. Gu, and N. Koshizuka, Phys. Rev. Lett. 75, 4516 (1995).

[86] S.-F. Lee, D. C. Morgan, R. J. Ormeno, D. Broun, R. A. Doyle, J. R. Waldram, and K. Kadowaki, Phys. Rev. Lett. 77, 735 (1996).

[87] A. Bhattacharya, I. Zutic, O. T. Valls, A. M. Goldman, U. Welp, and B. Veal, Phys. Rev. Lett. 82, 3132 (1999).

[88] M. Willemin, C. Rossel, J. Hofer, H. Keller, Z. F. Ren, and J. H. Wang, Phys. Rev. B 57, 6137 (1998).

[89] A. Sacuto, R. Combescot, N. Bontemps, P. Monod, V. Viallet, and D. Colson, Europhys. Lett. 39, 207 (1997).

[90] C. Kendziora, R. J. Kelley, and M. Onellion, Phys. Rev. Lett. 77, 727 (1996).

[91] J. Y. T. Wei, N.-C. Yeh, D. F. Garrigus, and M. Strasik, Phys. Rev. Lett. 81, 2542 (1998).

[92] C. C. Tsuei and J. R. Kirtley, Rev. Mod. Phys. 72, 969 (2000).

[93] Q. Li, Y. N. Tsay, M. Suenaga, R. A. Klemm, G. D. Gu, and N. Koshizuka, Phys. Rev. Lett. 83, 4160 (1999).

[94] A. G. Sun, D. A. Gajewski, M. B. Maple, and R. C. Dynes, Phys. Rev. Lett. 72, 2267 (1994).

[95] Z.-X. Shen et al., Phys. Rev. Lett. 70, 1553 (1993).

[96] H. Ding, J. C. Campuzano, A. F. Bellman, T. Yokoya, M. R. Norman, M. Randeria, T. Takahashi, H. Katayama-Yoshida, T. Mochiku, K. Kadowaki, and G. Jennings, Phys. Rev. Lett. 74, 2784 (1995).

[97] R. J. Kelley, C. Quitmann, M. Onellion, H. Berger, P. Almeras, and G. Margaritondo, Science 271, 1255 (1996).

[98] I. Vobornik, R. Gatt, T. Schmauder, B. Frazer, R. J. Kelley, C. Kendziora, M. Grioni, M. Onellion, and G. Margaritondo, Physica C 317-318, 589 (1999).

[99] Ya. G. Ponomarev et al., Physica C 243, 167 (1995).

[100] G. M. Zhao, Phys. Rev. B 64, 024503 (2001).

[101] G. M. Zhao, Phys. Rev. B 82, 012506 (2010).

[102] G. M. Zhao and J. Wang, J. Phys.: Condens. Matter 22, 352202 (2010).

[103] T. Das, R. S. Markiewicz, and A. Bansil, Phys. Rev. Lett. 98197004 (2007); Q. Yuan et. al., Phys. Rev. B 74214503 (2006).

[104] A. Garg, M. Randeria, and N. Trivedi, Nature Phys. 4, 762 (2008).

[105] C. C. Homes, R. P. S. M. Lobo, P. Fournier, A. Zimmers, and R. L. Greene, Phys. Rev. B 74, 214515 (2006).

[106] C. Panagopoulos, B.D. Rainford, J.R. Cooper, W. Lo, J.L. Tallon, J.W. Loram, J. Betouras, Y.S. Wang, and C.W. Chu, Phys. Rev. B 60, 14617 (1999).

[107] Y. Fukuzumi, K. Mizuhashi, K. Takenaka, and S. Uchida, Phys. Rev. Lett. 76, 684 (1996).

[108] J. Betouras and R. Joynt, Physica C 250, 256 (1995). 
[109] J. Mannhart and H. Hilgenkamp, Physica C 317-318, 383 (1999).

[110] A. S. Alexandrov, Physica C 305, 46 (1998).

[111] L. Alff, S. Meyer, S. Kleefisch, U. Schoop, A. Marx, H. Sato, M. Naito, and R. Gross, Phys. Rev. Lett. 83, 2644 (1999).

[112] Mun-Seog Kim, John A. Skinta, Thomas R. Lemberger, A. Tsukada, and M. Naito, Phys. Rev. Lett. 91, 087001 (2002).

[113] S. Kashiwaya, T. Ito, K. Oka, S. Ueno, H. Takashima, M. Koyanagi, Y. Tanaka, and K. Kajimura, Phys. Rev. B 57, 8680, (1998).

[114] Amlan Biswas, P. Fournier, M. M. Qazilbash, V. N. Smolyaninova, Hamza Balci, and R. L. Greene, Phys. Rev. Lett. 88, 207004 (2002).

[115] M. M. Qazilbash, Amlan Biswas, Y. Dagan, R. A. Ott, and R. L. Greene, Phys. Rev. B 68, 024502 (2003).
[116] L. Shan, Y. Huang, H. Gao, Y. Wang, S. L. Li, P. C. Dai, F. Zhou, J. W. Xiong, W. X. Ti, and H. H. Wen, Phys. Rev. B 72, 144506 (2005).

[117] L. Shan, Y. Huang, Y. L. Wang,S. l. Li, Jun Zhao, P. C. Dai, Y. Z. Zhang, C. Ren, and H. H. Wen, Phys. Rev. B 77, 014526 (2008).

[118] A. S. Alexandrov, Phys. Rev. B 77, 094502 (2008).

[119] Y. Yildirim and Wei Ku, cond-mat: arXiv:1005.0019

[120] Z. Radovic, N. Bozovic, and I. Bozovic, Phys. Rev. B 77, 092508 (2008); N. Gedik, D. S. Yang, G. Logvenov, I. Bozovic, A. H. Zewail, Science 316, 425 (2007).

[121] E. G. Maksimov, M. L. Kulic, and O. V. Dolgov, Adv. Condens. Matter Phys. 2010, 423725 (2010).

[122] J. Demsar, B. Podobnik, V. V. Kabanov, Th. Wolf, and D. Mihailovic, Phys. Rev. Lett. 82, 4918 (1999). 